\title{
Simple Methods to Approximate CPC Shape to Preserve Collection Efficiency
}

\author{
David Jafrancesco, Elisa Sani, Daniela Fontani, Luca Mercatelli, Paola Sansoni, \\ Annalisa Giannini, and Franco Francini
}

Italian National Institute of Optics, CNR-INO, Largo E. Fermi 6, 50125 Firenze, Italy

Correspondence should be addressed to Elisa Sani, elisa.sani@ino.it

Received 26 October 2011; Revised 9 December 2011; Accepted 15 December 2011

Academic Editor: Wayne A. Anderson

Copyright (C) 2012 David Jafrancesco et al. This is an open access article distributed under the Creative Commons Attribution License, which permits unrestricted use, distribution, and reproduction in any medium, provided the original work is properly cited.

The compound parabolic concentrator (CPC) is the most efficient reflective geometry to collect light to an exit port. Anyway, to allow its actual use in solar plants or photovoltaic concentration systems, a tradeoff between system efficiency and cost reduction, the two key issues for sunlight exploitation, must be found. In this work, we analyze various methods to model an approximated CPC aimed to be simpler and more cost-effective than the ideal one, as well as to preserve the system efficiency. The manufacturing easiness arises from the use of truncated conic surfaces only, which can be realized by cheap machining techniques. We compare different configurations on the basis of their collection efficiency, evaluated by means of nonsequential ray-tracing software. Moreover, due to the fact that some configurations are beam dependent and for a closer approximation of a real case, the input beam is simulated as nonsymmetric, with a nonconstant irradiance on the CPC internal surface.

\section{Introduction}

The interest in renewable energies, in particular in solar energy exploitation, is continuously increasing due to the combination of several facts, like the declining of fossil fuels, the growth of the world population, and the global climate change, as underlined by several authors (see, e.g., [1-3] and references therein). Solar power plants and in particular thermal and thermodynamic solar systems are receiving an increasing attention in the last years. They can exist in many possible architectures for a wide range of operating conditions and applications [4]. Among the available schemes, that of an heliostat field with bulk absorber cavity appears interesting for high-temperature large-scale plants because of its low heat loss, easy controlling, high heat capacity, and the possibility to successfully exploit mature technologies of conventional fossil fuel power plants [5]. As a general consideration, it should be noticed that in sunlight exploiting technologies, achieving the maximum efficiency is a key issue. When concentrating solar power (CSP) plants and solar furnace systems are considered, a relevant part of the efficiency of the overall system is due to the efficiency in light collection. Therefore, currently, there is a large interest in the development of techniques and methods to improve this facet. Heliostat fields often foresee a secondary light concentration apparatus for the efficient collection into the furnace of the radiation intercepted by the primary mirror system. Due to the fact that the receiver is generally angle insensitive and it is required to have a small entrance port to minimize radiation losses, a possible approach for the secondary mirror is to consider a compound parabolic concentrator (CPC), as this geometry allows, at least in 2D, reaching the theoretical limit for sunlight concentration $[6,7]$. In particular, CPCs can be very useful in large solar plants, where solar divergence prevents to obtain small focus areas, and, in high-temperature solar furnaces, where minimizing the entrance port dimensions is mandatory. The drawback of CPC is its parabolic surface, which is difficult and expensive to made and polish. In fact, the three-dimensional CPC is an axisymmetrical surface, obtained by the rotation of a parabola branch around an axis (that will be the axis of the obtained CPC), with the symmetry axis of the whole original parabola forming a nonzero 
angle with the $\mathrm{CPC}$ axis [8]. It is clear that this resulting complex surface arises in manufacturing problems and in high costs.

For this reason, different authors studied the possibility to truncate the CPC $[9,10]$ or to approximate CPCs by simpler shapes like planar, cylindrical or conical surfaces, or different combinations of them. In particular, authors in [11] report on the optimization of linear parabolic collectors (parabolic troughs) approximated by planar surfaces, while [12-14] are concerned with the approximation of CPCs with faceted surfaces, using simulations with generic light beams. Anyway, it should be noticed that the final result obtained from simulations will strongly depend on the characteristics of the considered input light beam. In fact a real beam produces an irradiation distribution on the CPC internal surface that is often nonhomogeneous in both longitudinal and radial directions. Such a distribution could be a disadvantage if we try to design a generic approximated CPC, but it is a strength point if we choose to lie within a beam-dependent approach. This latter has become now a real possibility thanks to the availability of nonsequential ray-tracing software, that allows to test many approximated CPC shapes taking in account the input beam. In this work, we analyze several CPC approximations, built using different strategies. As input beam for the analysis, we consider the beam produced from the simulation of a field of primary heliostats in a solar plant and we determine a general method to design a beamdependent approximated CPC. While the literature reports on many examples of optimization approaches, either using the edge-ray technique $[15,16]$ and specially designed elegant algorithms for a given system application (like, e.g., genetic algorithms $[17,18]$, hybrid evolutionary algorithms [19], Kiefer-Wolfowitz methods [11, 20]), to the best of our knowledge, simple methods allowing to easily use commercial software packages and at the same time both taking into account real world conditions and allowing to obtain a satisfying collection efficiency are lacking.

In the framework of studying systems aimed to be feasible and cost-effective, we decided to limit our investigation to approximated shapes which are cheap and easy to manufacture and polish, that is, truncated conic surfaces. Moreover, to keep costs and complexity as lower as possible, we analyzed a maximum number of 5 truncated cones per approximated CPC. It should be noticed that this also allows to reduce optical losses in the interconnection regions and to avoid possible assembly problems. Particular attention has been given to analyze the flux collected by the approximate surface, with the goal to identify the method that leads to define the most efficient shape among the analyzed ones. The quantitative evaluation of device performances and the comparison among the different approximated CPCs have been carried out using the Lambda Research TracePro software, a nonsequential lighting simulation package implementing MonteCarlo methods.

\section{Methods of CPC Approximations}

2.1. General. As said above, the simulation was performed considering a real beam coming from a primary mirror field.
In particular, this takes into account both the solar divergence and other contributions to the beam divergence, that is, heliostat pointing errors [21,22] and defects of the mirror surface. Solar divergence can be approximated as a uniform distribution with $4.7 \mathrm{mrad}$ half angle [23]; heliostat pointing errors arise in an almost uniform distribution with half angle depending on the characteristics of the Sun tracking system, but commonly evaluated to be about $3 \mathrm{mrad}$ [22]. It should be noticed that a uniform distribution with $b$ half angle can be approximated by a Gaussian distribution with $\sigma=b / \sqrt{ } 3$ standard deviation [24]. The estimation of slope local errors of mirror surfaces is more complex. We can make the hypothesis of a Gaussian distribution with $5 \mathrm{mrad}$ standard deviation [25]. Thus, the beam we choose for this work has Gaussian angular distribution of $\sigma=5.9 \mathrm{mrad}$, as resulting from the convolution of all the cited divergence sources. The rays of the beam have a maximum inclination of 0.39 rad with respect to the CPC axis.

All the simulations have been performed using 675000 rays. The statistical uncertainty connected with the MonteCarlo ray tracing is assessed tracing, for a given optical system under study, different sets of randomized rays. The maximum variation on the collected flux on the exit port of the optical system was $0.1 \%$.

The ideal starting CPC had input and output port of radii 301.8 and $115.5 \mathrm{~mm}$, respectively, $1007.5 \mathrm{~mm}$ total length and acceptance angle of $0.39 \mathrm{rad}$. The shape of every approximated CPC has to be a tradeoff between the request to keep the collection efficiency as high as possible and the need to be easy to realize and polish. A simple method is to utilize a series of truncated cones: the highest is the number of them, the more accurate is the approximation of the ideal surface. The advantage of this approach is that the approximated CPC maintains the radial curvature and the rotational symmetry, whereas the axial surface sections are planar. Obviously, each truncated cone is connected to the other, in such a way that the two extreme circumferences of each of them lie either on the entrance/exit port of the CPC or on an extreme circumference of the adjacent truncated cone. Moreover, due to the fact that, in the discussion, we will refer often to the longitudinal sections of the CPCs, in the following, we will label these circumferences as "points," because their projections on the longitudinal section appear as points.

To define the better (i.e., the most efficient) shape of the approximated CPC, we used four different approaches:

(i) $\mathrm{CPC1}$ (point-approximated $\mathrm{CPC}$ ): the approximated $\mathrm{CPC}$ is defined with an interpolation of the ideal CPC surface that does not take into account the irradiation data;

(ii) CPC2 (tangent-approximated CPC): we consider 4 or 5 conic sections with equally spaced angular coefficients of their longitudinal sections;

(iii) CPC3 (power-approximated CPC): the approximated surface takes into account the flux distribution on the ideal CPC internal surface;

(iv) $\mathrm{CPC} 4$ (corrected-power approximated CPC): the approximated surface takes into account both the flux 


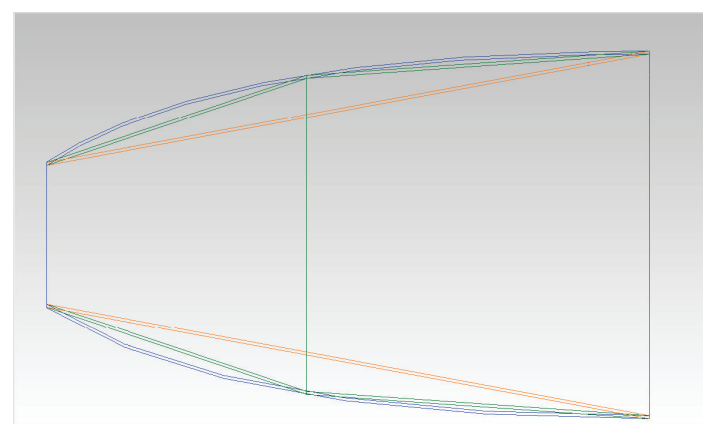

Figure 1: Ideal CPC (blue), 1-cone (orange) and 2-cone (green) point-approximated CPCs.

distribution on the ideal CPC internal surface and the effect of the difference, along the longitudinal section, between the approximated planar surface and the tangent plane to the ideal CPC.

We note in advance that the efficiency of the point-interpolation is quite low [12], but its very simple approximation method, described in the following, is a useful reference for most advanced approaches.

2.2. Point-Approximation Method. The method we used to build the point-interpolated CPC approximation is the following:

(1) the starting step is a 2-point approximation: a single truncated cone with the major and minor base circumferences equal to the entrance and exit aperture of the ideal CPC, respectively;

(2) the 3-point interpolation (2 truncated cones) has been built taking as connection point between the two cones the point on the ideal CPC where the distance between the ideal surface and the 2-point approximation is maximum;

(3) the approximation with $n$ truncated cones has been defined by dividing in two parts the truncated cone at the point where the distance between the internal surface of $(n-1)$-approximated CPC and those of the ideal CPC is maximum.

In Figures 1 and 2, we show the longitudinal sections of either the 1-cone and 2-cone (Figure 1) or 5-cone (Figure 2) approximated CPCs, compared to the ideal shape.

2.3. Tangent-Approximation Method. The tangent-approximated CPC has been obtained starting from the calculus of the derivative of the ideal CPC surface, for many points of the longitudinal section. Then, we evaluated the angular coefficient values of the tangent lines to the CPC border in the 2D longitudinal section at the input and output port. If we divide by 4 (for the 4 -cone approximation) or by 5 (for the 5 -cone approximation) the range of obtained angular coefficients, we find the step in the angular coefficient values between adjacent lines, for 4-cone or 5-cone approximations, respectively. The successive step is to identify the angular coefficient

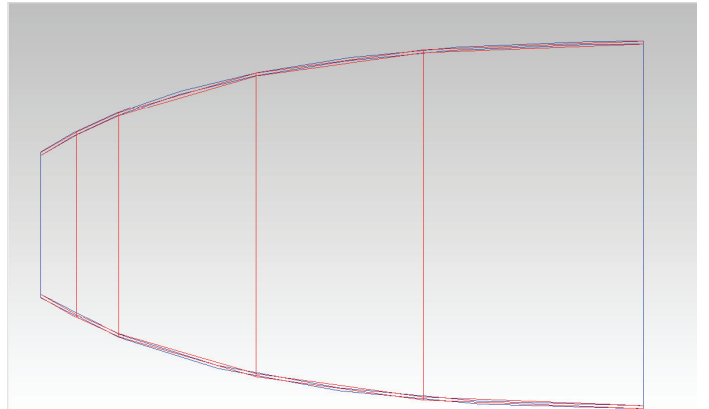

FIgURE 2: Ideal CPC (blue) and 5-cone (red) point-approximated CPC.

of each line and the point of the ideal CPC being related to this coefficient. Starting from these points, we draw the tangent lines. As for the peculiar lines lying at the input and output ports, we translate them to match the port diameter. Then, we determine the intersection points, whose 3D-corresponding circumferences will be the bases of 3D-truncated cones. It should be noticed that, in 2D, the cones are represented as segments of tangent lines. Finally, the merging of all truncated cone shapes produces the tangent-approximated $3 \mathrm{D}$ solid. Please note that, in this approach, it is geometrically impossible to model a 2- or 3-cone approximation.

2.4. Power-Approximation Method. This is a beam-dependent approach: the starting step is to consider the irradiance distribution on the internal surface of the ideal CPC, as produced by the actual beam coming from the heliostat field.

Figure 3 shows the projection of irradiance values for the whole CPC surface on a plane parallel to the CPC axis, while Figure 4 represents the irradiance profiles obtained from Figure 3 along the $y=0$ and $x=0$ directions. It should be noticed that, being Figure 3 a projection of values, the irradiance value of a single pixel lying on the axis in Figure 3 represents twice the mean value of the irradiance on the corresponding ring of the 3D ideal CPC shown in Figure 4. Starting from Figures 3 and 4, we calculate the power (i.e., the radiant flux) on each ring, then the integrated power as a function of the coordinate along the ideal CPC axis, obtaining the picture shown in Figure 5. The interpolating point has been chosen in such a way that each truncated cone equally contributes to the overall power integral (i.e.: in case of 5 truncated cones, each contributes for $20 \%$ to the total incident power).

2.5. Corrected Power-Approximation Method. In this case, we introduced a correction factor to apply to the previously described power-approximation approach. It is intuitive that the same small slope variation produces a larger effect on the collected power when the surface is far from the exit aperture of the CPC. Then, we can introduce a weight parameter to correct the calculus of the power-approximation method. This parameter is calculated in a fully geometrical way, measuring, in different points of the $\mathrm{CPC}$, the displacement of the reflected ray due to an error of $1^{\circ}$ on the slope of the surface. Figure 6 shows the considered geometry. The displacement is 


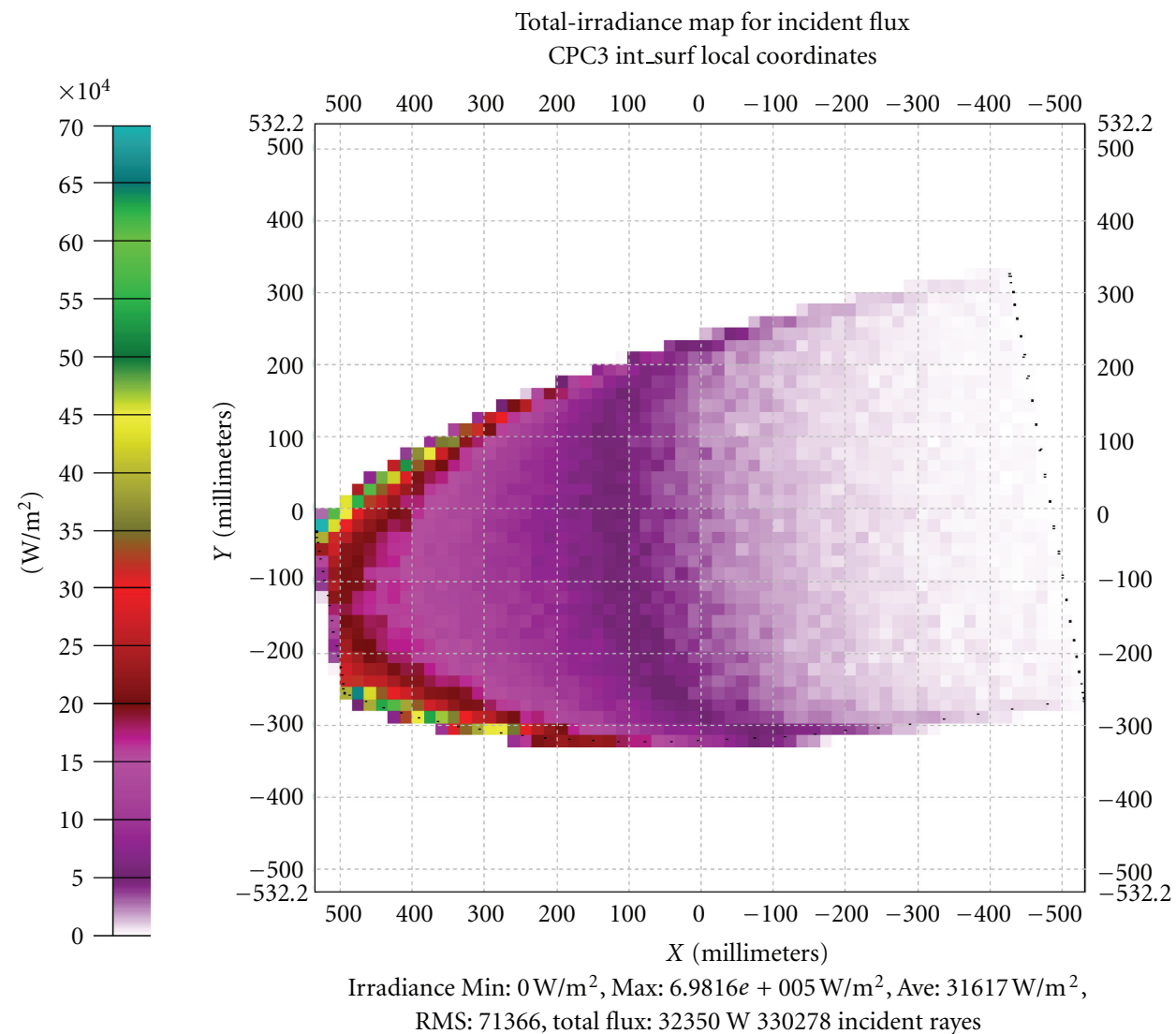

FIgUre 3: Projected irradiance map of the ideal CPC.

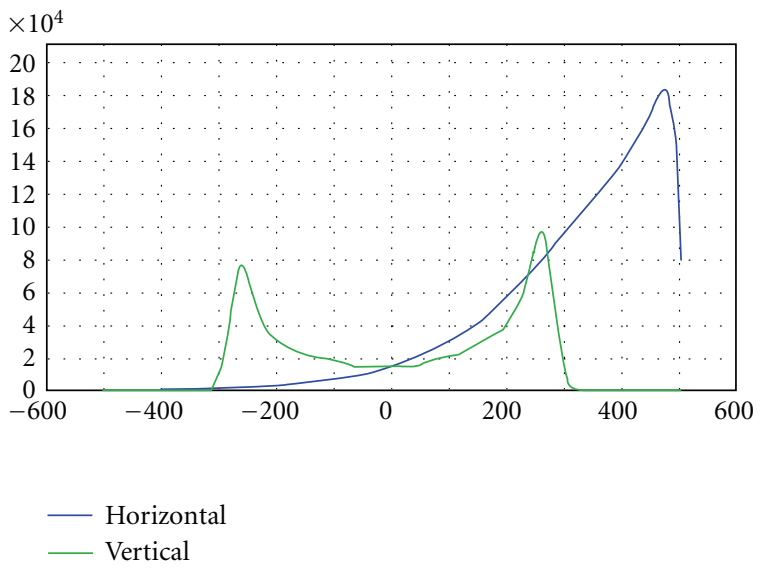

Figure 4: Irradiance profiles on a longitudinal plane parallel to the axis of the ideal CPC. Blue line: irradiance profile along the $y=0$ direction of the in the irradiance map. Green line: irradiance profile along the $x=0$ direction of the in the irradiance map.

evaluated on the exit port of the CPC, in relative units with respect to the port dimension itself. It quantifies the surface sensitivity, at the considered point, to angular errors of the surface due to surface approximations. From this tolerance analysis, we obtained a correction function that, after normalization to its maximum value (corresponding to surface

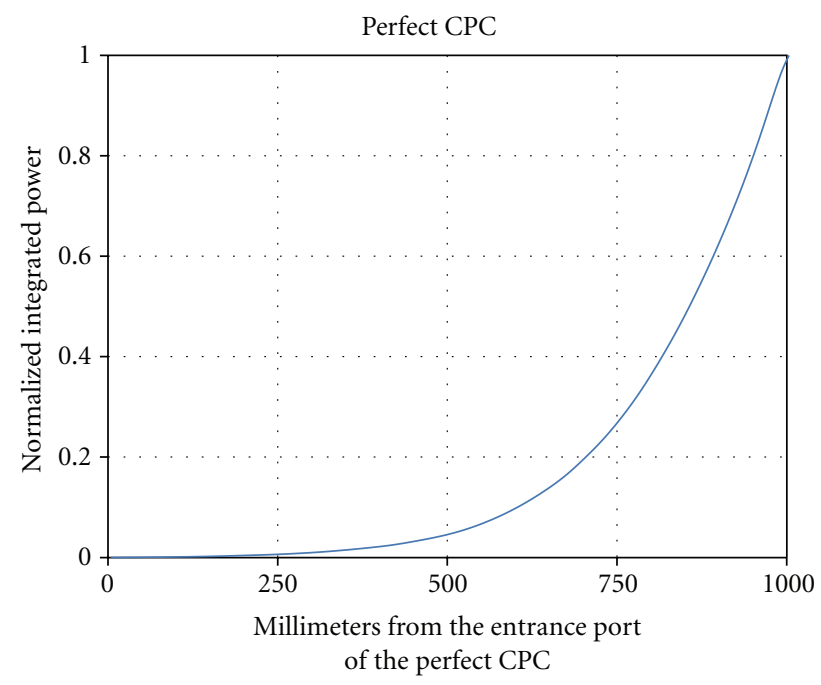

FIGURE 5: Normalized integral power incident on a small longitudinal section of the ideal CPC coplanar to the CPC axis.

points near the entrance of the $\mathrm{CPC}$ ), was used to correct the integral in Figure 5. In Figure 7, we compare the integral functions we obtained using the corrected-power approximation method to that produced by the simple power one described in the previous section. Similarly to Section 2.4, 


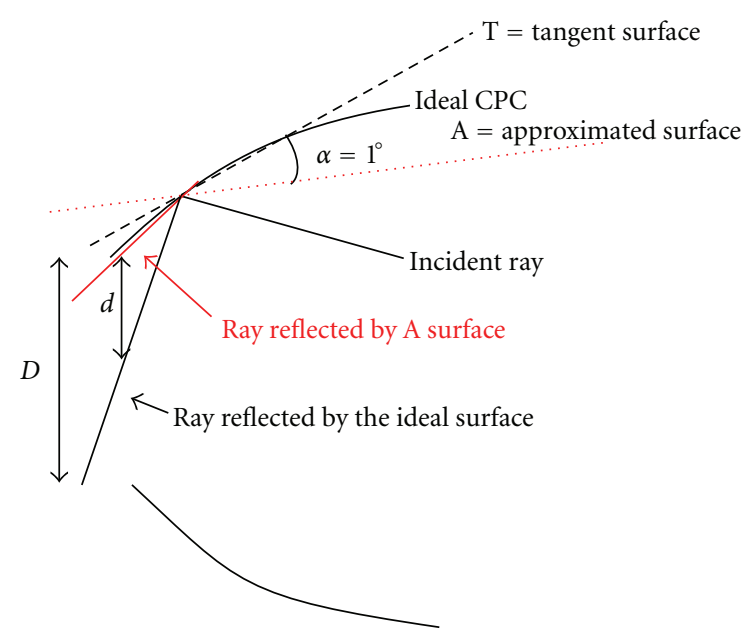

FIGURE 6: Geometry used in the corrected-power approximation method. $D$ : diameter of the CPC exit port; $d$ : distance of the two reflected rays measured on the exit port. The quantity relevant for the method is the relative displacement $d / D$. Drawing is exaggerated for better readability.

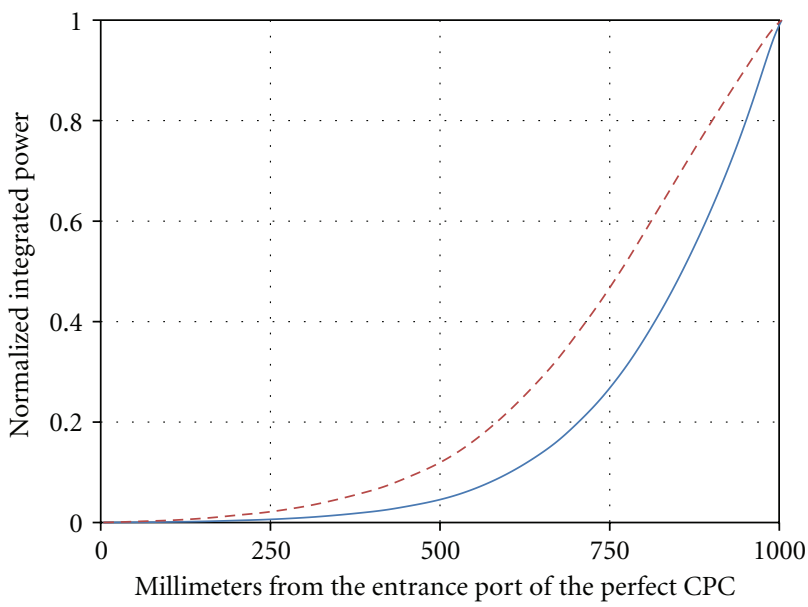

FIGURE 7: Comparison between simple power-approximation method (solid line) and corrected-power approximation method (dashed line). The lines refer to the normalized integral power incident on a small longitudinal section of the ideal CPC coplanar to the $\mathrm{CPC}$ axis (solid line) and to the same integral function after correction according to the corrected-power approximation method (dashed line).

dimensions of truncated cones to approximate the ideal CPC are obtained imposing that each cone equally contributes to the overall integral. From Figure 7, we can appreciate that the corrected-power method, for fixed number of cones, requires that the cones near the entrance aperture of the approximated CPC are shorter than those calculated with the simple corrected power method, thus correctly taking into account the larger sensitivity to angular surface errors of the approximated $\mathrm{CPC}$ rings lying near the entrance port.

Figure 8 shows the comparison among different 5 -cone CPCs. All the approximated CPCs have been positioned to make their entrance and exit apertures coincident with the
TABLE 1: Simulation results in terms of collected power at the output port. The flux collected by the ideal surface is also reported for reference.

\begin{tabular}{|c|c|c|c|c|c|}
\hline \multirow[b]{2}{*}{ Type } & \multicolumn{5}{|c|}{$\%$ Collected power } \\
\hline & $\begin{array}{c}1- \\
\text { section }\end{array}$ & $\begin{array}{c}2- \\
\text { section }\end{array}$ & $\begin{array}{c}3- \\
\text { section }\end{array}$ & $\begin{array}{c}4- \\
\text { section }\end{array}$ & $\begin{array}{c}5- \\
\text { section }\end{array}$ \\
\hline Ideal & & & 100 & & \\
\hline Point approximation & 93.7 & 79.4 & 91.9 & 94.5 & 95.4 \\
\hline $\begin{array}{l}\text { Tangent } \\
\text { approximation }\end{array}$ & & & & 97.4 & 97.9 \\
\hline Power approximation & & 93.0 & 96.0 & 97.4 & 97.7 \\
\hline $\begin{array}{l}\text { Corrected-power } \\
\text { approximation }\end{array}$ & & 87.9 & 94.7 & 97.3 & 98.7 \\
\hline
\end{tabular}

entrance and exit aperture of the ideal CPC. In Figure 8(a), we compare the point-approximated (green), the tangentapproximated (orange), and the power-approximated (blue) ones, while Figure 8(b) compares the power-approximated (blue) to the corrected power-approximated (red). We note that, while the point-approximated and the power-approximated CPCs are similar if the positions of the interpolation points are concerned, both power-approximated CPCs provide a finer approximation of the region near the exit aperture, where the majority of the incident power is collected.

\section{Results}

The results of the performed simulations are summarized in Table 1 and in Figure 9. We can see that, except for the 2-cone case of the point-approximated CPC, the output power increases as the number of section increases. The counterintuitive case of the 2-cone point-approximated CPC, that shows a minimum of output power, agrees with the literature [12]. The reason of the nonmonotonic dependence of the collected flux from the number of considered cones could be that the value of the slope of the single-cone approximation is near to the tangent to the ideal CPC at the point of maximum irradiance.

If we compare different approximation approaches for equal number of considered cones, we can observe that the efficiency of the point-approximated CPC is the lowest. The power and the tangent approximations produce very similar results. The corrected-power approximation shows a lower efficiency than the simple power approach for the 2- and 3cone configurations, but a higher efficiency for the 5-cone one. For the 4-cone case, tangent, power, and correctedpower approximations practically lead to the same result.

The best result is obtained for the 5-cone corrected-power approximated CPC: it has efficiency as high as $98.7 \%$ with respect to that of the ideal CPC. We stress that we imposed the same constraints in the simulations for all the CPCs: first, CPC ports of fixed dimensions; second, length of the approximated CPCs equal to the length of the ideal CPC; third, reflectivity of all the surfaces set to $85 \%$. In particular, this latter is consistent with the actual value of reflectance of 


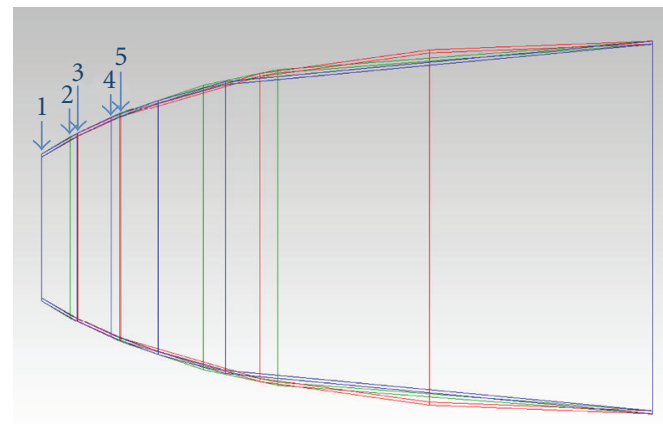

(a)

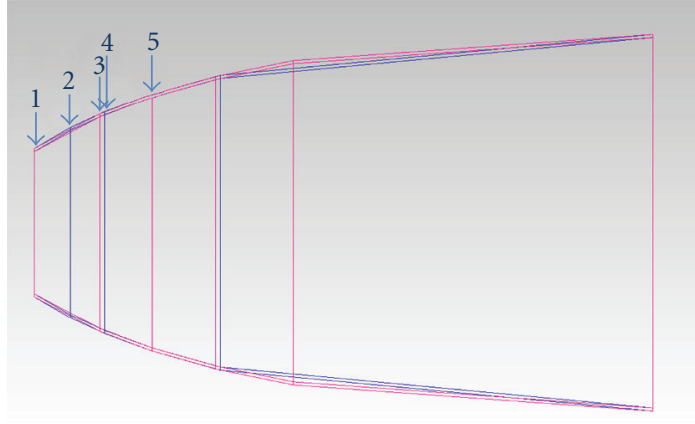

(b)

Figure 8: (a) Point-approximated CPC (red), tangent-approximated CPC (green), and power-approximated CPC (blue). Superimposed vertical lines are present at positions corresponding to labels 3 (red and blue) and 5 (green and red). (b) Power-approximated CPC (blue) and corrected power-approximated CPC (pink). Superimposed blue and pink vertical lines are present at the position corresponding to label 5 .

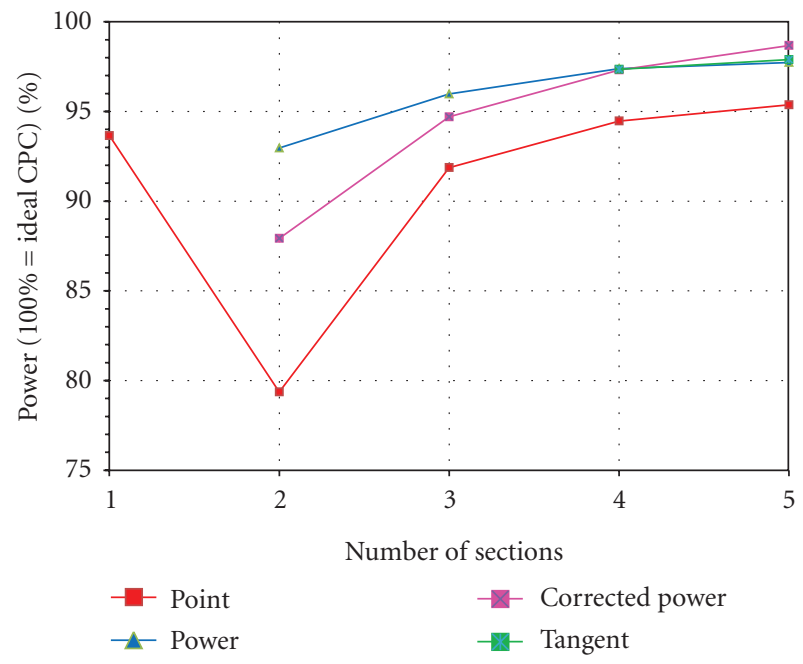

FIGURE 9: collected power versus number of sections for pointapproximated (green), tangent-approximated (orange), power-approximated (blue), and corrected power-approximated CPC (red).

a coating of protected aluminum, thus further projecting our results towards practically realizable systems.

\section{Conclusions}

In this work, we study the possibility to approximate the complex shape of a large compound parabolic concentrator by means of a limited number of truncated cones, demonstrating that this is feasible without severe efficiency losses. We investigated different approximation approaches and with variable number of truncated cones. Keeping in mind, beside the efficiency preservation, also the practical realization of the system and the manufacturing and cost decrease, we limited our study to a maximum number of 5 cones. We demonstrated that the most successful approach is to link the geometrical surface approximation to the irradiance distribution on the internal surface of the ideal CPC, as produced by the real available beam (beam-dependent scheme).
With 5 considered cones, the shape showing the largest collection efficiency is a corrected-power approximated CPC, producing efficiency as high as $98.7 \%$ with respect to the ideal CPC. The advantage of power-approximation and corrected power-approximation methods versus other approaches such as the tangent one is the possibility to easy implement also 2-cone or 3-cone geometries. We found that the collected power is minimum for the 2-cone point interpolation, evidencing a nonmonotonic behavior of the collected power as a function of the number of sections. Finally, we remark that the beam-dependent methods we propose can be successfully applied also when the irradiance distribution on the internal surface of the CPC changes during the day or during the year, and when the time-averaged distribution results strongly nonhomogeneous.

\section{References}

[1] J. A. Turner, "A realizable renewable energy future," Science, vol. 285, no. 5428, pp. 687-689, 1999.

[2] R. Shinnar and F. Citro, "Solar thermal energy: the forgotten energy source," Technology in Society, vol. 29, no. 3, pp. 261$270,2007$.

[3] Q. Liu, Q. Miao, J. J. Liu, and W. Yang, "Solar and wind energy resources and prediction," Journal of Renewable and Sustainable Energy, vol. 1, Article ID 043105, 12 pages, 2009.

[4] S. A. Kalogirou, "Solar thermal collectors and applications," Progress in Energy and Combustion Science, vol. 30, no. 3, pp. 231-295, 2004.

[5] M. Romero, R. Buck, and J. E. Pacheco, "An update on solar central receiver systems, projects, and technologies," Journal of Solar Energy Engineering, vol. 124, no. 2, pp. 98-108, 2002.

[6] W. T. Welford and R. Winston, The Optics of Nonimaging Concentrators, Academic Press, New York, NY, USA, 1st edition, 1978.

[7] H. Ries, "Thermodynamic limitations of the concentration of electromagnetic radiation," Journal of the Optical Society of America, vol. 72, no. 3, pp. 380-385, 1982.

[8] R. Winston, J. C. Miñano, and P. Benítez, Nonimaging Optics, Elsevier Academic Press, San Diego, Calif, USA, 1st edition, 2005. 
[9] A. Rabl, "Optical and thermal properties of compound parabolic concentrators," Solar Energy, vol. 18, no. 6, pp. 497-511, 1976.

[10] M. J. Carvalho, M. Collares-Pereira, J. M. Gordon, and A. Rabl, "Truncation of CPC solar collectors and its effect on energy collection," Solar Energy, vol. 35, no. 5, pp. 393-399, 1985.

[11] A. J. Marston, K. J. Daun, and M. R. Collins, "Geometric optimization of concentrating solar collectors using monte carlo simulation," Journal of Solar Energy Engineering, vol. 132, no. 4, Article ID 041002, 2010.

[12] A. Timinger, A. Kribus, P. Doron, and H. Ries, "Faceted concentrators optimized for homogeneous radiation," Applied Optics, vol. 39, no. 7, pp. 1152-1158, 2000.

[13] T. H. Roos, Y. Maharajh, and D. J. Griffith, "Design philosophy and construction of a high concentration compound parabolic concentrator," in Proceedings of the Solar Power and Chemical Energy Systems (SolarPACES '10), pp. 21-24, Perpignan, France, September 2010.

[14] A. Segal, "Approximated secondary CPC, built of planar facets, adjustable for two solar central receivers," in Optical Materials Technology for Energy Efficiency and Solar Energy Conversion XIII, V. Wittwer, C. G. Granqvist, and C. M. Lampert, Eds., vol. 2255 of Proceedings of SPIE, pp. 567-572, April 1994.

[15] R. Leutz, A. Suzuki, A. Akisawa, and T. Kashiwagi, "Design of a nonimaging Fresnel lens for solar concentrators," Solar Energy, vol. 65, no. 6, pp. 379-387, 1999.

[16] W. Spirkl, H. Ries, J. Muschaweck, and A. Timinger, "Optimized compact secondary reflectors for parabolic troughs with tubular absorbers," Solar Energy, vol. 61, no. 3, pp. 153$158,1997$.

[17] J. Beaulieu, C. Gagné, and M. Parizeau, "Lens system design and re-engineering with evolutionary algorithms," Proceedings of the Genetic and Evolutionary Computation Conference (GECCO '02), pp. 155-162, 2002.

[18] P. Kotsidas, E. Chatzi, and V. Modi, "Stationary nonimaging lenses for solar concentration," Applied Optics, vol. 49, no. 27, pp. 5183-5191, 2010.

[19] N. Yamada and T. Nishikawa, "Evolutionary algorithm for optimization of nonimaging Fresnel lens geometry," Optics Express, vol. 18, supplement 2, pp. A126-A132, 2010.

[20] K. J. Daun, D. P. Morton, and J. R. Howell, "Geometric optimization of radiant enclosures containing specular surfaces," Journal of Heat Transfer, vol. 125, no. 5, pp. 845-851, 2003.

[21] R. O. Hughes, "Effects of tracking errors on the performance of point focusing solar collectors," Solar Energy, vol. 24, no. 1, pp. 83-92, 1980.

[22] V. Badescu, "Theoretical derivation of heliostat tracking errors distribution," Solar Energy, vol. 82, no. 12, pp. 1192-1197, 2008.

[23] D. Buie, A. G. Monger, and C. J. Dey, "Sunshape distributions for terrestrial solar simulations," Solar Energy, vol. 74, no. 2, pp. 113-122, 2003.

[24] European Pre-standard "Guide to the expression of uncertainty in measurements", CEN ENV 13005, 1999.

[25] J. O'Gallagher and R. Winston, "Performance model for twostage optical concentrators for solar thermal applications," Solar Energy, vol. 41, no. 4, pp. 319-325, 1988. 


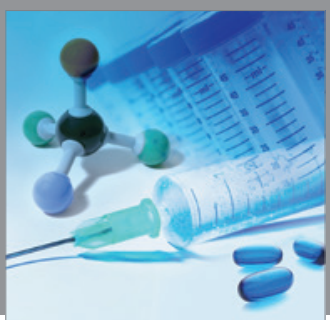

International Journal of

Medicinal Chemistry

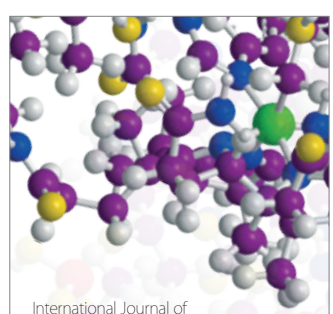

Carbohydrate Chemistry

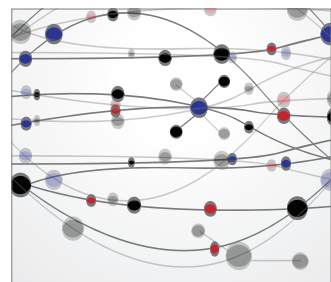

The Scientific World Journal
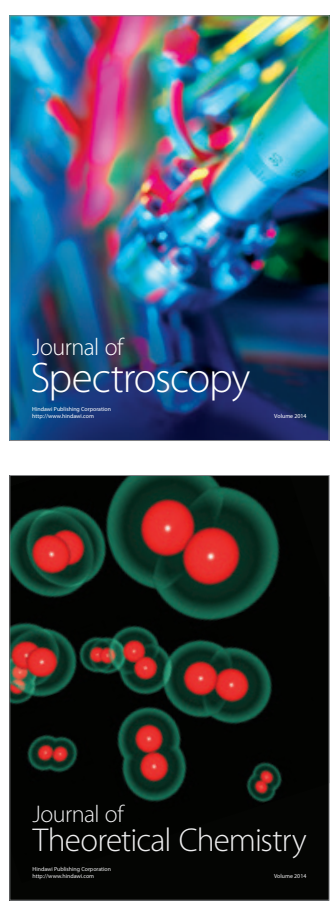
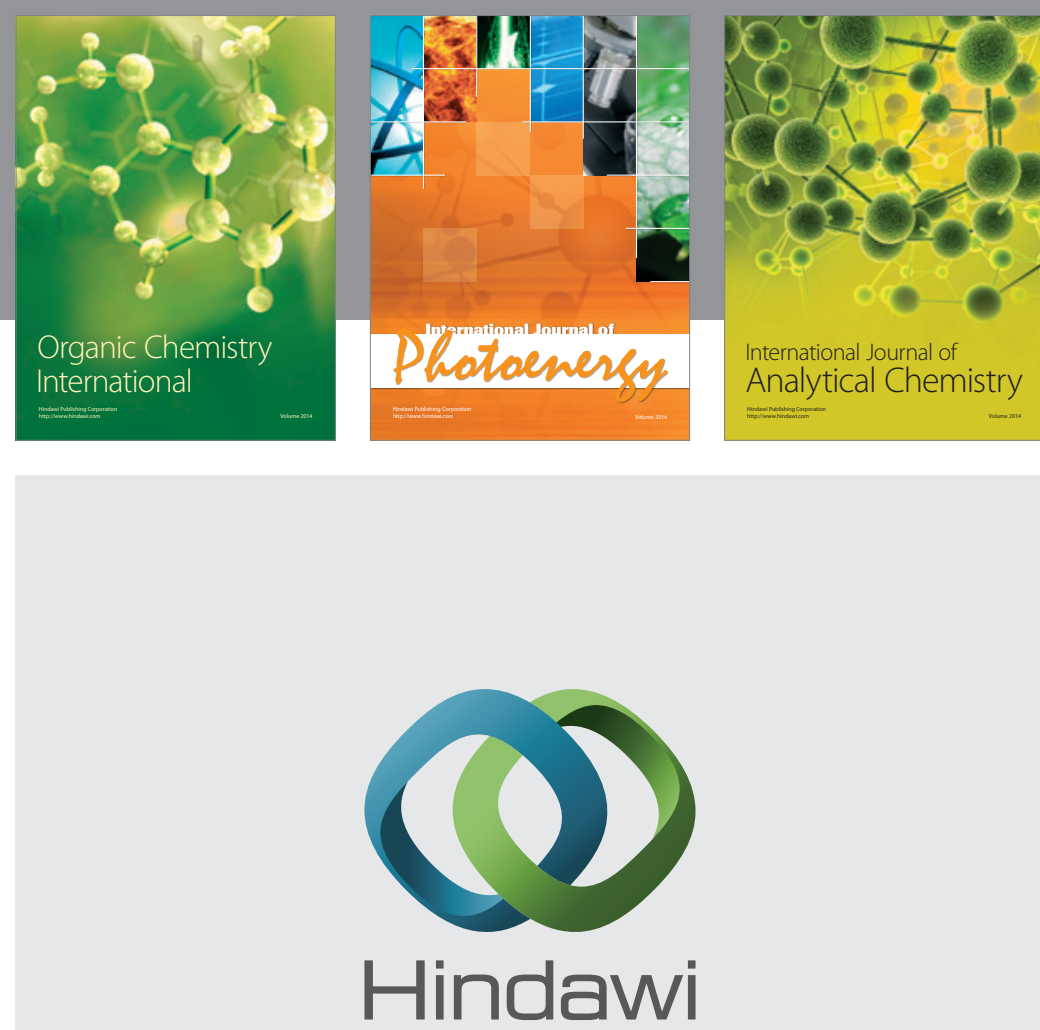

Submit your manuscripts at

http://www.hindawi.com
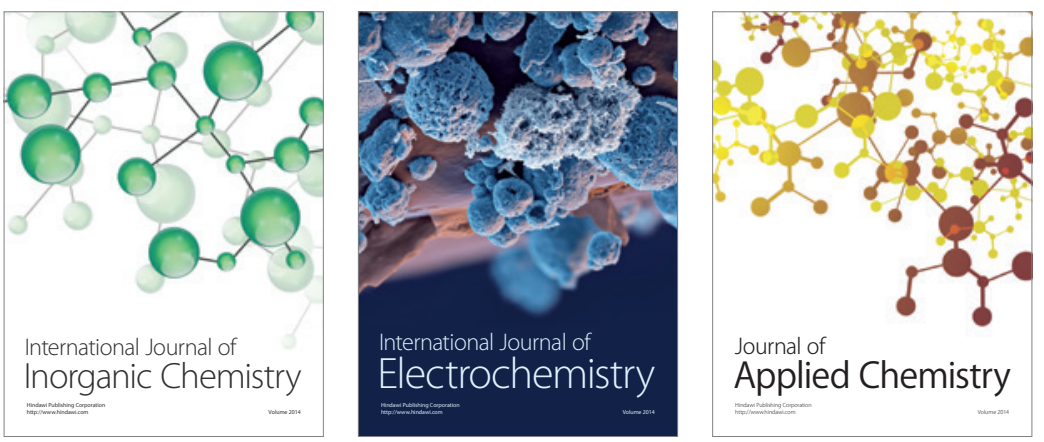

Journal of

Applied Chemistry
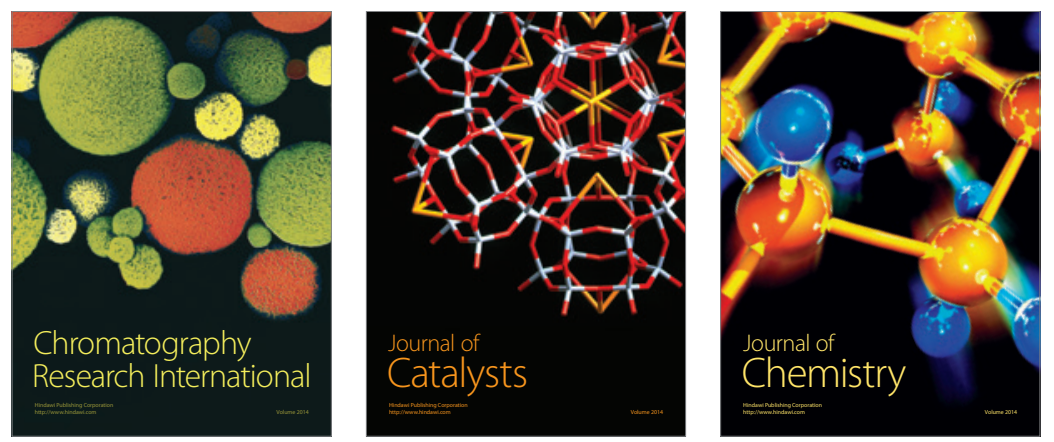
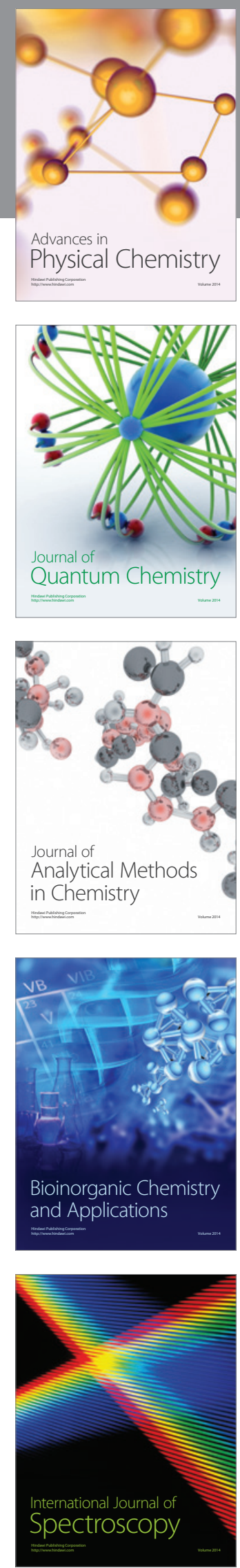